5th International Workshop on Astronomy and

Relativistic Astrophysics (IWARA2011)

International Journal of Modern Physics: Conference Series

Vol. 18 (2012) 86-90

(C) World Scientific Publishing Company

DOI: $10.1142 / \mathrm{S} 2010194512008252$

\title{
ON THE GEOMETRICAL CONFINEMENT OF FERMIONS
}

\author{
J. B. FORMIGA \\ Departamento de Fsica, Universidade Federal de Fsica, \\ C. Postal 5008, 58051-970 Joo Pessoa, Pb, Brazil \\ jansen@fisica.ufpb.br \\ C. ROMERO \\ Departamento de Fsica, Universidade Federal de Fsica, \\ C. Postal 5008, 58051-970 Joo Pessoa, Pb, Brazil \\ cromero@fisica.ufpb.br
}

\begin{abstract}
To show the important role that geometry may play in the problem of confining particles to our four-dimensional spacetime, we consider a five-dimensional model where fermions are confined in a hypersurface due to an interaction with geometric fields. We use two different fields which appear in non-Riemannian geometries, namely, the Weyl one-form and the torsion two-form. We show that for suitable choices of these fields one manages to confine fermions in our brane (our four-dimensional world). It turns out that this confinement is independent of the energy and the mass of the fermions.
\end{abstract}

Keywords: geometry; torsion; non-metricity; confinement; fermions.

PACS numbers: 02.40.-k, 04.60.-m, 04.50.-h

\section{Introduction}

Many models of modern physics is based on the assumption that the spacetime has more than four dimensions ${ }^{1}$. However, we feel the world as if it were a fourdimensional spacetime. When dealing with compact extra dimensions this can be explained through the assumption that their compactification radii are very small, which makes them hard to be detected. Nonetheless, in a model with large extra dimensions the particles could get out of our brane and disappear forever. In 1983, Rubakov brought light to this problem by creating a model where massless neutrinos stay inside the brane when their energy is $l_{0}{ }^{2}$. His model is endowed with a scalar field, which is responsible for confining the massless fermions, and a five-dimensional Minkowski spacetime.

Our goal is to show how geometry can play a important role in the problem of confinement. To do that, we use two non-Riemannian geometries: Riemann-Cartan geometry and a geometry characterized by a tensor called non-metricity, which we refer to as "non-metricity geometry". In both cases, we will use the minimal coupling 
procedure in the field equations (MCPE). This article is organized as follows. In Sec. 2 we present our notation and conventions, while in Sec. 3 we give a very brief introduction to non-Riemannian geometries. Sec. 4 is devoted to the two possible Dirac equations, while Secs. 5 and 6 are dedicated to the confinement of fermions. Some final remarks are given in Sec. 7 .

\section{Notation and Conventions}

\subsection{Vielbein formalism}

We use the set $\left\{\theta^{A}\right\}$ to represent the vielbein one-form, while the frames will be represented by $\left\{e_{A}\right\}$. A vielbein basis is defined as being a basis in which the metric tensor written in terms of it coincides with the Minkowski metric, that is, $g\left(e_{A}, e_{B}\right)=\eta_{A B}$, where $\eta_{A B}=\operatorname{diag}(1,-1,-1, . .,-1)$. We refer to both $\left\{\theta^{A}\right\}$ and $\left\{e_{A}\right\}$ as vielbein.

\subsubsection{Affine connection in a vielbein basis}

The use of the same symbol to represent the affine connection in a different basis may be a little bit misleading, since this connection is not a tensor. So we shall use $\omega^{A}{ }_{B C}$ to represent the affine connection in the vielbein basis, but the others will be denoted by $\Gamma$. Our definition of the components of the affine connection is $\Gamma^{\dot{a}}{ }_{\dot{b} \dot{c}} \equiv<e^{\dot{a}}, \nabla_{e_{\dot{b}}} e_{\dot{c}}>$, where the over dot is just to make clear that the letters do not represent any particular type of index, that is, this is the affine connection in an arbitrary basis $\left\{e_{\dot{a}}\right\}$ and its dual $\left\{e^{\dot{a}}\right\}$. Now, it is important to set our index notation. Greek letters stand for coordinate indexes, capital Latin letters represent vielbein indexes, and small Latin letters are spin indexes.

\subsection{Spinor notation}

The Dirac spinor will be denoted by $\Psi$, which represents a column matrix whose elements are $\Psi^{a}$. The complex conjugate transpose of $\Psi$ is $\Psi^{\dagger}$, which is a row matrix whose elements are denoted by $\Psi_{a}$. The Dirac matrices are represented by $\gamma^{\mu}$, which in the vielbein basis coincide with the well-known Dirac matrices in Minkowski spacetime. As usual we use $\bar{\Psi}=\Psi^{\dagger} \gamma^{(0)}$, where the parentheses surrounding the zero is to indicate that this gamma is calculated in the vielbein basis.

\subsubsection{Covariant derivative}

The components of the covariant derivative of the spinors $\Psi$ and $\bar{\Psi}$ are $\Psi_{\mid \mu}=$ $\Psi_{, \mu}+\Gamma_{\mu} \Psi$ and $\bar{\Psi}_{\mid \mu}=\bar{\Psi}_{, \mu}-\bar{\Psi} \Gamma_{\mu}$, respectively. The symbol $\Gamma_{\mu}$ denotes the spin connection (sometimes called "internal connection") and can be written in the form

$$
\Gamma_{\alpha}=A_{\alpha} \mathbb{I}+\frac{1}{8} \omega_{A \alpha B}\left[\gamma^{A}, \gamma^{B}\right]
$$


if we demand $\gamma_{\mid \alpha}^{\mu}=0$ (see, e.g., Ref. 3), where $A_{\mu}$ is the components of a tensor and $\mathbb{I}$ is the unit matrix. When non-metricity is present we have to change this condition. Nonetheless, the form of the spin connection remains the same. Note that in (1) we have ${ }^{\mathrm{a}} \omega_{A \alpha B} \equiv \eta_{A C} e^{D}{ }_{\alpha} \omega^{C}{ }_{D B}$, where $e^{D}$ are the components of the vielbein in a coordinate basis.

\section{Non-Riemannian Geometries}

There are many different kinds of geometries that generalize the Riemannian one ${ }^{4-6}$. The most common examples are those which generalize the affine connection ${ }^{\mathrm{b}}$ : Riemann-Cartan geometry and non-metricity geometries. In what follows we focus on the affine connection generalization.

\subsection{Riemann-Cartan geometry}

Riemann-Cartan geometry is characterized by a tensor called torsion $\mathcal{T}(V, U)$, which is defined as

$$
\mathcal{T}(V, U) \equiv \nabla_{V} U-\nabla_{U} V-[V, U],
$$

where $V, U$ are vectors belonging to the tangent plane of the Riemann-Cartan manifold $\mathbb{U}$, and $\nabla$ is the affine connection. The torsion tensor is identically zero in a Riemannian manifold.

We define the components of the torsion in an arbitrary basis $\left\{e_{\dot{a}}\right\}$ as $T_{\dot{b} \dot{c}}^{\dot{a}} \equiv<$ $d x^{\dot{a}}, \mathcal{T}\left(\partial_{\dot{b}}, \partial_{\dot{c}}\right)>$, while the components of the contorsion tensor are defined as $K^{\dot{a}}{ }_{\dot{b} \dot{c}} \equiv-\left(T_{\dot{b} \dot{c}}{ }^{\dot{a}}+T_{\dot{c} \dot{b}}{ }^{\dot{a}}-T^{\dot{a}}{ }_{\dot{b} \dot{c}}\right) / 2$.

\subsection{Non-metricity geometries}

The non-metricity geometries $\mathbb{W}$ are characterized by the non-metricity tensor $\mathcal{N}$, which is defined by

$$
\mathcal{N}(V, U, W) \equiv \nabla_{W} g(V, U),
$$

where $V, U, W$ are vectors, and $g$ is the metric tensor. In a coordinate basis, we have $\stackrel{N}{\Gamma}_{\mu \nu}^{\lambda}=\stackrel{c}{\Gamma}_{\mu \nu}^{\lambda}+g^{\lambda \sigma}\left(-N_{\sigma \mu \nu}-N_{\nu \sigma \mu}+N_{\mu \nu \sigma}\right) / 2$, where $\stackrel{c}{\Gamma}_{\mu \nu}^{\lambda}$ is the Christoffel symbol.

For consistency with (3), we have to change the condition $\gamma_{\mid \alpha}^{\mu}=0$ for $\gamma_{\mid \lambda}^{\mu}=$ $-N^{\mu}{ }_{\nu \lambda} \gamma^{\nu} / 2$

\subsubsection{Weyl geometry}

Weyl geometry is a particular case of the non-metricity geometries. In this geometry, we have $\mathcal{N}(V, U, W)=\sigma(W) g(V, U)$, where $\sigma(W)$ is the Weyl one-form.

\footnotetext{
${ }^{a}$ We are using Einstein's summation convention

${ }^{\mathrm{b}}$ Though less common in the literature, geometries which generalize other aspects of Riemann geometry have also been taken into account ${ }^{7,8}$.
} 


\subsubsection{Riemann-Cartan plus Weyl geometry}

If both torsion and non-metricity are present, then $\Gamma_{\mu \nu}^{\lambda}=\stackrel{c}{\Gamma}_{\mu \nu}^{\lambda}+\stackrel{n}{\Gamma}_{\mu \nu}^{\lambda}+K_{\mu \nu}^{\lambda}$, where $\stackrel{n}{\Gamma}_{\mu \nu}^{\lambda} \equiv g^{\lambda \sigma}\left(-N_{\sigma \mu \nu}-N_{\nu \sigma \mu}+N_{\mu \nu \sigma}\right) / 2$, with $N_{\mu \nu \alpha}=\sigma_{\alpha} g_{\mu \nu}$.

\section{Minimal Coupling Procedure and the Dirac Equation}

Roughly speaking, the minimal coupling procedure (MCP) means the following changes $\partial_{\mu} \rightarrow \nabla_{\mu}, \eta_{\mu \nu} \rightarrow g_{\mu \nu}, V=$ constant $\rightarrow V=V(x), d x^{\mu} \rightarrow \sqrt{-g} d x^{\mu}$. In a Riemannian geometry there is no ambiguity in this procedure. However, in a non-Riemannian one we have to decide whether we apply it in the field equations or in the Lagrangian: the MCP applied to the Lagrangian (MCPL) yields field equations that are different from those obtained when one applies the MCP to the field equations directly. As a result there are two versions of the Dirac equation, as shown below.

By applying the MCPE we obtain

$$
i \gamma^{\mu}\left(\partial_{\mu}+\Gamma_{\mu}\right) \Psi-m \Psi=0
$$

while the MCPL yields

$$
i \gamma^{\mu}\left[\partial_{\mu}+\Gamma_{\mu}+\frac{1}{2} N_{[\lambda \mu]}^{\lambda}+\frac{1}{2} T_{\mu \lambda}^{\lambda}\right] \Psi-m \Psi=0 .
$$

\section{Confinement via Weyl Field}

For Weyl geometry, a straightforward calculation leads Eq. (4) to

$$
i \gamma^{\alpha}\left(\partial_{\alpha}+\stackrel{c}{\Gamma}_{\alpha}-\frac{n-1}{4} \sigma_{\alpha}\right) \Psi-m \Psi=0,
$$

where $n$ is the spacetime dimension, $\stackrel{c}{\Gamma}$ is the part of the spin connection that does not depend on $\sigma$ (the "Riemannian part"), and we have set $A_{\mu}=0$.

Let us now assume that the spacetime has five dimensions, consider the representation of the Dirac matrices as being those of four dimensions, and take the fifth Dirac matrix as $-\gamma^{(0)} \gamma^{(1)} \gamma^{(2)} \gamma^{(3)}$, as usual. Taking $\sigma=d \phi$ and making the substitution $\Psi=\psi e^{\phi(l)}$, where $l$ is the coordinate of the fifth dimension, we arrive at

$$
i \gamma^{\alpha}\left(\partial_{\alpha}+\stackrel{c}{\Gamma}_{\alpha}\right) \psi-m \psi=0 .
$$

If we admit that $\lim _{l \rightarrow \pm \infty} \psi<e^{l^{2}}$, then a convenient choice for $\phi$ ensures the confinement. As an example, we may take $\phi=-l^{2}$. In this case the fermions will be trapped to the brane $l=0$. 


\section{Confinement via Torsion}

If we take $\sigma=0, T_{\beta \mu}^{\alpha}=f(l)_{, \nu}\left(\delta_{\beta}^{\alpha} \delta_{\mu}^{\nu}-\delta_{\mu}^{\alpha} \delta_{\beta}^{\nu}\right)$ and consider a five-dimensional spacetime, the Eq. (4) will become

$$
i \gamma^{\mu}\left(\partial_{\mu}+\stackrel{c}{\Gamma}_{\mu}\right) \Psi+2 i f_{, \nu} \gamma^{\nu} \Psi-m \Psi=0 .
$$

By making the substitution $\Psi=\psi(x) e^{-2 f(l)}$, we get Eq. (7) again. Therefore, we can procedure as before to ensure the confinement.

It is clear that we can easily generalize this procedure to $n$ dimension and confine the fermions in any brane we wish. See Ref. 9 for more details.

\section{Final Remarks}

To analyze the confinement with the MCPL, we have to use Eq. (5). However, the method applied in the previous sections does not work in this case. So we limit ourselves to the following statements:

- Scalar fields cannot be confined through either torsion or non-metricity directly, since in the MCPL they do not feel these fields ${ }^{10}$.

- Massless spinor fields satisfying the Weyl condition $\left(1+\gamma^{5}\right) \Psi / 2=\Psi$ cannot be confined via torsion directly, since $K_{C A B} \gamma^{[A} \gamma^{B} \gamma^{C]}=0$ (see, e.g., p. 263 of Ref. 11).

- We are also unable to confine fermions via non-metricity directly because Eq. (5) does not depend on $N_{\mu \nu \lambda}$.

\section{Acknowledgments}

C. Romero and J. B. Formiga would like to thank CNPq for financial support.

\section{References}

1. V. A. Rubakov, Phys. Usp. 44, 871 (2001).

2. V. A. Rubakov and M. E. Shaposhnikov, Phys. Lett. B125, 136 (1983).

3. J. P. Crawford, Class. Quant. Grav. 20, 2945 (2003).

4. R. T. Hammond, Rept. Prog. Phys. 65, 599 (2002).

5. D. Puetzfeld, New Astron. Rev. 49, 59 (2005).

6. A. H. Fariborz and D. G. C. McKeon, Class. Quant. Grav. 14, 2517 (1997).

7. T. Damour, S. Deser, and J. G. McCarthy, Phys. Rev. D47, 1541 (1993).

8. D. Sen and J. Vanstone, J. Math. Phys. 13, 990 (1972).

9. C. Romero, J. B. Formiga, and C. Dariescu, Grav. Cosmol. 17, 252 (2011).

10. D. Puetzfeld, Y. N. Obukhov, Phys. Rev. D76, 084025 (2007).

11. V. de Sabbata and M. Gasperini, Introduction to Gravitation. (World Scientific, Singapore, 1985). 